\title{
Non-Invasive Ventilation (NIV): Failure is Not a Four-Letter Word
}

Rob Graham, R.R.T./N.R.C.P.

I dedicate this column to the late Dr. Andrew (Andy) Shennan, the founder of the perinatal program at Women's College Hospital (now at Sunnybrook Health Sciences Centre). To my teacher, my mentor and the man I owe my career as it is to, thank you. You have earned your place where there are no hospitals and no NICUs, where all the babies do is laugh and giggle and sleep.

"Not so long ago, premature babies were intubated and ventilated strictly based on gestational age (GA) and not a clinical condition. Many of these babies ended up with chronic lung disease (CLD) that could have likely been avoided had they not been intubated."

NIV may be the most substantial change in neonatal ventilation since neonatal ventilators were invented. Before ventilators, NIV was the only respiratory support available to clinicians caring for neonates. As the saying goes, "everything old is new again." The concept may be old, but the practice is quite clearly here to stay. That is not a bad thing. Not so long ago, premature babies were intubated and ventilated strictly based on gestational age (GA) and not a clinical condition. Many of these babies ended up with chronic lung disease (CLD) that could have likely been avoided had they not been intubated.

The tools available to clinicians have changed considerably during neonatology's relatively short life. While bubble CPAP and nasopharyngeal tubes (NPTs) were once the only NIV game in town, today we have a myriad of equipment and modalities at our disposal as well as different interfaces (I confess to being fondly reminiscent of NPTs).

In addition to having a greater selection of equipment to choose from, we now also have several NIV modes available. I have used non-invasive positive pressure ventilation (NIPPV) in my practice since I started as a respiratory therapist over 32 years ago. Back in the day, it was referred to as "NPT puffs," and pressures were set by occluding the circuit and adjusting accordingly, and standard settings were a peak inspiratory pressure (PIP) of $12 \mathrm{cmH}_{2} \mathrm{O}$ and a PEEP of $5 \mathrm{cmH}_{2} \mathrm{O}$ at a rate of 12 . The practice was laughed at by other NICUs in Toronto, but fast forward to today, and its use is commonplace, albeit with different interfaces.

Without leak compensation and adaptive flow, older machines delivered considerably less pressure than was set. Combined with the low PEEP/CPAP level, this undoubtedly resulted in many failures and subsequent intubation/reintubation. A major difference today is that newer ventilators are more adept at maintaining and delivering pressure than those of old, and pressures are set while the baby is connected; thus, the baby receives what is dialed in.

Other NIV modalities include high flow (HF) and non-invasive high-frequency oscillation (NIHFO), and both of these modes have been used extensively over the last decade or so. The latter may improve the odds of successful extubations (1). I have previously written on non-invasive high-frequency jet ventilation (coined "NINJA"), and while some research has been done on the topic $(2,3)$, it has not (as of yet) seen utilisation in the clinical setting. Until the FDA approval of $3^{\text {rd }}$ generation ventilators with NIHFO capability, this is the only high-frequency NIV mode currently available to American clinicians.

\section{"The newest adjunct to NIV is the use of neurally adjusted ventilatory assist (NIV- NAVA), which allows for synchronization of NIPPV as well as assessment of the adequacy of CPAP level through diaphragmatic tone."}

The newest adjunct to NIV is the use of neurally adjusted ventilatory assist (NIV-NAVA), which allows for synchronization of NIPPV as well as assessment of the adequacy of CPAP level through diaphragmatic tone. NIV-NAVA shows great promise in facilitating successful extubation and more efficient NIV, possibly by decreasing work of breathing (WOB) and by decreasing the amount of gastric air in NIPPV through synchronization (4). It is rapidly gaining favour for the provision of NIV.

Technical improvements in NIV have undoubtedly increased the likelihood of success, while the patients we place on NIV have gotten ever smaller. Simultaneously, "ETTphobia" threatens to overtake "PEEPaphobia" as the dominant malady in NICUs as NIV has been taken up with religious fervor around the world. This is not a problem when NIV is used judiciously on appropriate patients who indeed do not require intubation and are best served by an NIV modality; the problem arises when it is not.

NEONATOLOGY TODAY is interested in publishing manuscripts from Neonatologists, Fellows, NNPs and those involved in caring for neonates on case studies, research results, hospital news, meeting announcements, and other pertinent topics.

Please submit your manuscript to: LomaLindaPublishingCompany@gmail.com 
As a practicing bedside clinician, I have always believed in treating the patient, not the numbers. NIV can and should be considered even for a tiny baby should that baby's respiratory drive be sufficient and oxygen requirements low enough, but as much thought should be put into failure criteria as is put into the selection of respiratory support. Like an emotional investor who watches their money disappear when the value of the stock they "believe in" plummets into oblivion, too many clinicians stubbornly refuse to intubate a baby who is telling them loudly and clearly in the only language available to them that they are failing. What is that language? There are several dialects: FiO2, apnea, bradycardia, and WOB.

\section{$\mathrm{FiO}_{2}$}

July's NT column discussed $\mathrm{O}_{2}$ and $\mathrm{O}_{2}$ toxicity and how they relate to gestational age and endogenous antioxidant protection (or lack thereof). The relative lack of antioxidant protection in the micropremature infant is well known. What is now becoming clearer are the consequences of long-term exposure to increased levels of $\mathrm{O}_{2}$ : impaired pulmonary function later in life and structural lung abnormalities (5), not to mention retinal damage. This effect appears to be a combination of $\mathrm{O}_{2}$ concentration and time, and it has been suggested that cumulative $\mathrm{O}_{2}$ exposure during the first 72 hours of life is predictive of "respiratory symptoms and respiratory-related health service and medication use during infancy in a dose-dependent manner" (5).

\section{"While high FiO2 is detrimental over the long term, it is wise to avoid it during the first 72 hours of ventilatory management as much as possible."}

While high $\mathrm{FiO}_{2}$ is detrimental over the long term, it is wise to avoid it during the first 72 hours of ventilatory management as much as possible. I maintain that the closest one can manage a baby to $21 \% \mathrm{O}_{2}$, the better.

Finally, increasing $\mathrm{O}_{2}$ requirements are usually an indication that the lungs are de-recruiting. Ignored, this may progress to total collapse with associated atelectrauma and resulting inflammatory cascade.

\section{Apnea and/or Bradycardia}

Apneic and bradycardic episodes are de rigueur in the premature infant even with adequate "caffeination." NIPPV or NIHFO may mitigate the severity of spells but come with the risk of increasing gastric air. The changes in $\mathrm{SpO}_{2}$ and cerebral blood flow associated with these episodes can contribute to the development of ROP and cerebral bleeds, particularly during the first 72 hours of life. An infant requiring frequent stimulation and adjustments in $\mathrm{FiO}_{2}$ during this period may be better off being intubated and ventilated with a lung-protective strategy until this risk period is over.

\section{WOB}

The smallest babies will generally display a degree of WOB, and because their chest walls lack rigidity, some degree of intercostal indrawing is usually evident. In and of itself, this is not of great importance unless quite severe; however, when the chest wall significantly retracts, this represents a problem. This may appear to be more severe than is actually the case in the presence of a pectus excavatum, as the concave chest gives the appearance of retraction even at rest. Severe indrawing is evidence of high airway resistance and/or low pulmonary compliance. It may be accompanied by expiratory grunting, the infant's physiological response to maintain airway patency on expiration. This demonstrates the need for greater support than is being provided to the baby.

Tachypnea is often cited as evidence that an infant is experiencing increased WOB. Faster breathing clearly requires more energy but smaller babies (just like smaller animals) tend to have faster respiratory rates than larger ones. In isolation, mild tachypnea is not concerning; however, in combination with other clinical observations such as retractions and increasing $\mathrm{FiO}_{2}$, it is. Tachypnea can be a sign of insufficient recruitment since insufficient functional residual capacity (FRC) results in decreased pulmonary compliance. In this case, drawing smaller breaths at a faster rate requires less energy than breathing at a slower rate with larger tidal volumes. Note that as compliance deteriorates, further grunting may ensue, and respiratory rate may decrease as the collapsing lung increases airway resistance $(\mathrm{Ra} / \mathrm{w})$ which, in turn, requires longer expiratory time.

\section{"Tachypnea can be a sign of insufficient recruitment since insufficient functional residual capacity (FRC) results in decreased pulmonary compliance."}

\section{Modes and Devices}

\section{Stand Alone NIV Devices}

Bubble CPAP (BCPAP)

The bubbling from bubble (BCPAP) produces a high frequency, low amplitude oscillation, and noise that may explain its history of reliability and successful use. Even with this relatively ancient, simple modality, some factors influence both the frequency and amplitude of the bubble BCPAP, such as the flow rate, the diameter of the submerged tube and the diameter of the BCPAP bottle (6). Changing the underwater portion of the circuit also has implications with a "J" shape improving oscillatory amplitude and consistency (7), and the noise generated by the bubbling water itself may also be implicated as a contributing factor to the mode's success (8). BCPAP may be used with virtually any NIV interface that will fit.

\section{Stand Alone NIV devices}

The first commercial device that provided CPAP was the "Aladdin ${ }^{\circledR \text { " }}$ from Hamilton Medical, later renamed the Infant Flow System ${ }^{\circledR}$. This device came with proprietary securement hats, nasal prongs, and mask interfaces. A fluidic flip in the interface was purported to reduce WOB by reducing expiratory resistance though some question the validity of the evidence supporting this. Later models (called SiPAP ${ }^{\circledR}$ ) included providing bi-level pressure at an adjustable rate and inspiratory time. Later, an interface for synchronization was added; however, it did not work very well in practice. The Achilles heel of this system was its "safety" pressure limit. Once a system pressure of $12 \mathrm{cmH}_{2} \mathrm{O}$ was reached, it would dump all pressure for a few seconds. This happened more frequently as support pressures increased such that, in practice, a CPAP level of $8 \mathrm{cmH}_{2} \mathrm{O}$ was its practical operational limit. Given that this most commonly occurred when treating patients needing a high level of support and who were on the verge of requiring intubation it was, to say the least, frustrating. 
High Flow Nasal Canulae (HF)

$\mathrm{HF}$ is one of the simplest ways to deliver CPAP. Nasal cannulae are connected to a humidified source of gas flow, and the flow rate is adjusted to deliver desired support. The cannulae must be capable of delivering high enough flow rates. The RAM ${ }^{\circledR}$ canulae are likely the most widely used interface for HF delivery. The Wilkinson formula (9) has been used to estimate delivered CPAP pressure, but many units simply adjust the flow rate to clinical effect in practice. Many infants respond quite well to this modality, and an added benefit is patient comfort since the prongs do not have to be tightly fitted for effect.

\section{"he RAM® canulae are likely the most} widely used interface for HF delivery. The Wilkinson formula (9) has been used to estimate delivered CPAP pressure, but many units simply adjust the flow rate to clinical effect in practice."

\section{Ventilators}

Early neonatal ventilators such as the Sechrist ${ }^{\circledR}$ were used to provide CPAP (and NIPPV) via a nasopharyngeal tube (NPT). This is a regular ETT cut to a length either as determined by direct laryngoscopy to sit just behind the soft palate, or, as was the practice in the unit I practice in, just over $4 \mathrm{~cm}$. (The "P" of Portex on a Portex ETT). With no leak compensation or synchronization, this was rudimentary but did allow for a backup rate and inspiratory time (Ti). A problem associated with these systems is how pressure was typically set: circuit was occluded, pressure(s) set, and the device attached to the NPT. Delivered pressure(s) were invariably lower than set, compounded by CPAP levels of $5 \mathrm{cmH}_{2} \mathrm{O}$ were rarely exceeded. In addition, the resistance of the NPT increased WOB, especially with a longer length.

Third-generation ventilators now include NIV modes. Leak compensation, when available, greatly improves the consistency of delivered pressure in the presence of air leak but in NIPPV mode, maintaining peak inspiratory pressure remains a challenge. Decreasing the slope or increasing inspiratory flow (if inspiratory and expiratory flows can be adjusted independently) may help. If HFO mode is available, these machines may also be used to deliver NIHFO, although the mode has not been validated. Some machines can also provide "oxygen therapy," a constant flow delivered through the inspiratory limb of the circuit that can be used for HF. This eliminates the need for additional equipment and circuits (other than the NIV interface); however, backpressure from nasal prongs may cause the machine to dump pressure. (I have performed bench testing that seemed to indicate $12 \mathrm{lpm}$ was a maximum flow rate with the Drager ${ }^{\circledR} \mathrm{VN} 500$, although I have seen the machine dumping pressure at lower flows).

\section{NIV Failure}

The surest way to fail our patients on NIV is by failing to establish what represents failure. May I suggest several?

$\mathrm{FiO}_{2}$

Increasing $\mathrm{O}_{2}$ requirements usually indicate pulmonary derecruitment. Increasing CPAP support or use of early NIPPV may reverse or mitigate increased $\mathrm{FiO}_{2}$; however, once derecruitment occurs, re-recruitment is very difficult using NIV. In addition, lungs are very prone to damage during recruitment on top of the damage caused by derecruitment. I suggest an increase of $>10 \%$ in $\mathrm{O}_{2}$ requirements should put NIV failure on the radar, combined with other criteria doubly so.

Leaks

Even the most modern NIV devices and interfaces are rendered less effective in the face of leaks, be they around the interface itself or from an open mouth. Chin straps may be used to reduce leakage from the mouth (and may be necessary with higher support pressures), and interfaces should be snug but not tight (with the exception of the $\mathrm{RAM}^{\circledR}$ canulae). Nasal prongs should be sized such that they just occlude the nares, and the nasal septum should be visible and not blanched. Nasal masks are sometimes better tolerated, but care must also be taken to avoid undue pressure on the nasal bridge and upper lip. Overtightening of nasal prongs can result in catastrophic damage to the nasal septum, whereas with nasal masks, the bridge of the nose can be excoriated, or the entire centre of the face may be caved in.

Pressure and the Law of Diminishing Return

As NIV support pressure increases, so does the amount of gastric air. Ensuring oral gastric tubes are in situ and vented to air helps, as does routine aspiration of air from the stomach. Eventually, even with due diligence, there may be so much air accumulation in the stomach that it compromises lung volume. This results in increased WOB, tachypnea, and eventually complete derecruitment. From a practical bedside perspective, I find CPAP pressures or NIVHFO MAP of $\geq 12 \mathrm{cmH}_{2} \mathrm{O}$ are likely to result in excessive gastric distention and/or nasal damage from efforts to maintain pressure if used for an extended period. As such, the requirement for this level of pressure should be considered a failure.

\section{"As NIV support pressure increases, so does the amount of gastric air. Ensuring oral gastric tubes are in situ and vented to air helps, as does routine aspiration of air from the stomach. Eventually, even with due diligence, there may be so much air accumulation in the stomach that it compromises lung volume."}

\section{PEEPAPHOBIA}

I have bolded this sub-heading because it may be the factor most likely to lead to NIV failure. Infants extubated to NIV should be placed on a CPAP level equal to the MAP prior to extubations, not PEEP. This may be weaned as indicated, but failure to provide sufficient distending pressure post-extubation is a sure path to failure. Similarly, when NIV is the first intention, pressure must be adequate to aid in recruitment. Initially, this may be $10-15$ $\mathrm{cmH}_{2} \mathrm{O}$ or more. PEEPaphobia on the admission table is likely to buy the patient an ETT.

Other Considerations

The need to vent gastric air as well as feed necessitates the placement of an OGT. In some infants, the existence of an OGT may 
decrease vagal tone leading to bradycardic or apneic episodes. One might be tempted to place the tube nasally to avoid this when problematic. This should not be done as notching the nare from pressure required to maintain a seal can occur quickly with an NIV interface.

"One might be tempted to place the tube nasally to avoid this when problematic. This should not be done as notching the nare from pressure required to maintain a seal can occur quickly with an NIV interface."

\section{Summary}

NIV is a universally accepted form of respiratory support in the NICU, and its use will only increase as technology improves. In our desire to avoid the pitfalls of intubation and mechanical ventilation, failing to recognize that NIV is not meeting the patient's needs is not the patient's failure; it's the clinician's.

\section{References:}

1. https://pubmed.ncbi.n/m.nih.gov/31414062/

2. https://clinicaltrials.gov/ct2/show/NCT03558737

3. https://clinicaltrials.gov/ct2/show/NCT03558737

4. https://bmcpediatr.biomedcentral.com/articles/10.1186/ s12887-019-1683-4

5. $\quad$ https://err.ersjournals.com/content/27/147/170108

6. https://www.researchgate.net/publication/234018837

7. https://www. seattlechildrens.org/research/centers-programs/integrative-brain-research/our-labs/neonatal-respiratory-support-technologies-team/sea-pap/

8. https://www.nature.com/articles/pr2005154

9. Wilkinson formula for HF: pharyngeal pressure $(\mathrm{cm}$ $\left.\mathrm{H}_{2} \mathrm{O}\right)=0.7+1.1 \mathrm{~F}\left(\mathrm{~F}=\right.$ flow per $\mathrm{kg}$ in $\left./ \mathrm{min}^{-1} \mathrm{~kg}^{-1}\right)$

Disclosures: The author receives compensation from Bunnell Inc for teaching and training users of the LifePulse HFJV in Canada. He is not involved in sales or marketing of the device nor does he receive more than per diem compensation. Also, while the author practices within Sunnybrook H.S.C. this paper should not be construed as Sunnybrook policy per se. This article contains elements considered "off label" as well as maneuvers, which may sometimes be very effective but come with inherent risks. As with any therapy, the riskbenefit ratio must be carefully considered before they are initiated.

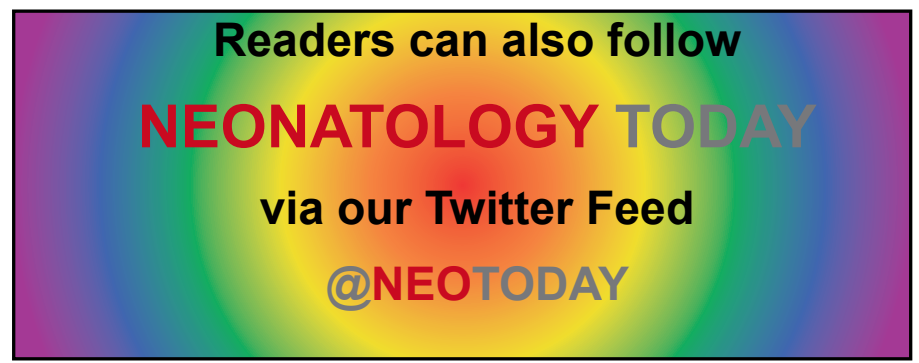

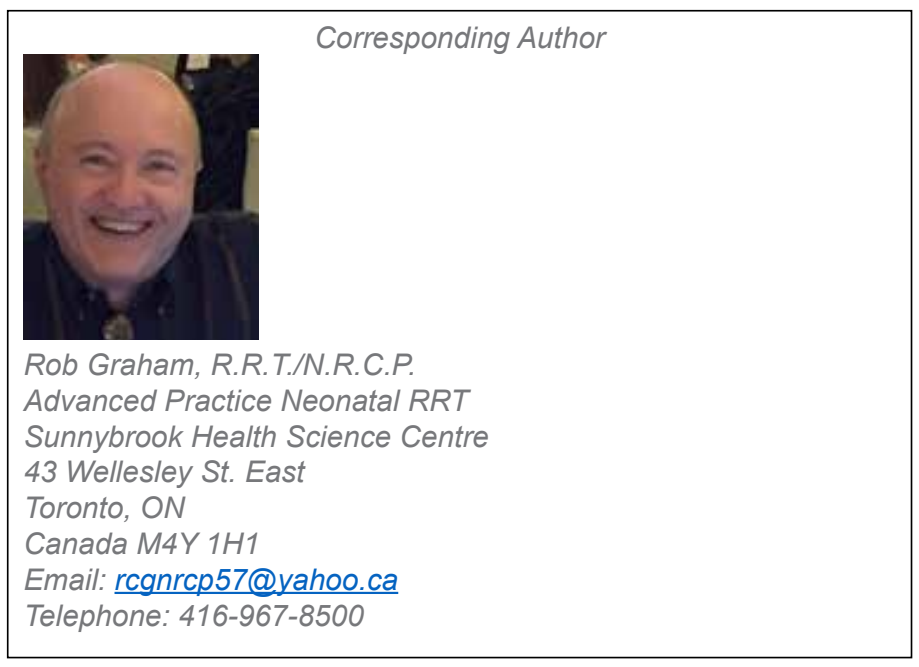

\section{O National Perinatal Association PERINATAL MENTAL HEALTH}

nationalperinatal.org/position

www.nationalperinatal.org/mental_health

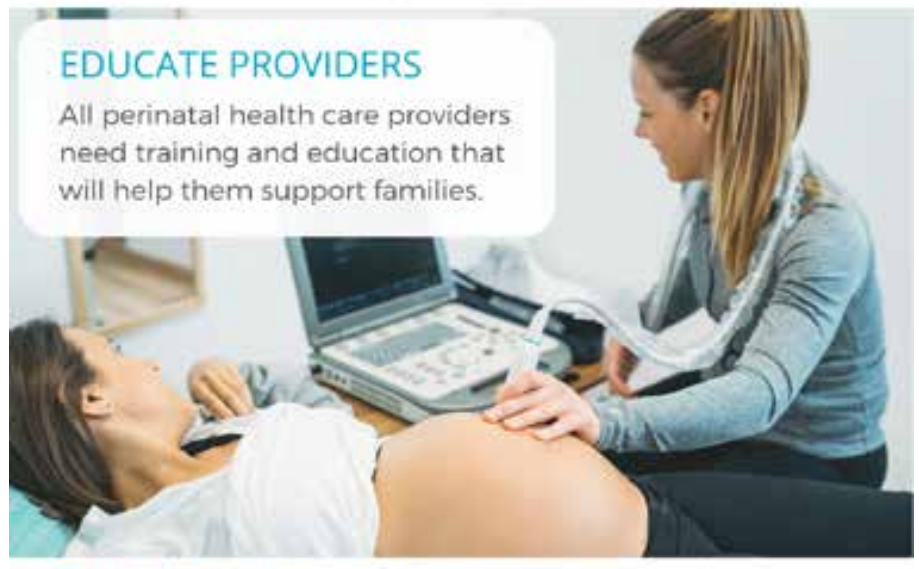

Educate. Advocate. Integrate.

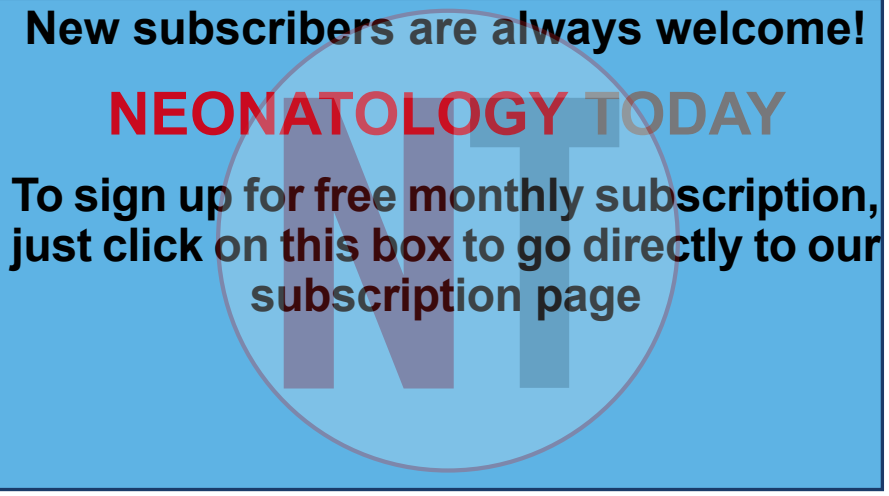

